SHORT REPORT

\title{
Tourette's syndrome and deep brain stimulation
}

\author{
J L Houeto, C Karachi, L Mallet, B Pillon, J Yelnik, V Mesnage, M L Welter, S Navarro, A Pelissolo, \\ P Damier, B Pidoux, D Dormont, P Cornu, Y Agid
}

In this prospective double blind randomised " $\mathrm{N}$ of 1 " study, a patient with a severe form of Tourette's syndrome was treated with bilateral high frequency stimulation of the centromedian-parafascicular complex (Ce-Pf) of the thalamus, the internal part of the globus pallidus (GPi), or both. Stimulation of either target improved tic severity by $70 \%$, markedly ameliorated coprolalia, and eliminated self injuries. Severe forms of Tourette's syndrome may benefit from stimulation of neuronal circuits within the basal ganglia, thus confirming the role of the dysfunction of limbic striatopallido-thalamo-cortical systems in this disorder.

$\mathrm{T}$ ourette's syndrome is characterised by motor and vocal tics associated with self injurious behaviours and other psychiatric manifestations that affect social and professional life. ${ }^{1}$ Severe forms of the disease respond poorly to medical treatment, which has potentially serious side effects. ${ }^{2}$ Severe tics have been improved by median thalamotomy ${ }^{3}$ and hyperkinesias by pallidotomy, ${ }^{4}$ but brain lesioning can cause severe irreversible adverse reactions. ${ }^{5}$ Continuous high frequency stimulation, a reversible neurosurgical method, ${ }^{6}$ has positive effects on tics when the median thalamus is targeted, ${ }^{7}$ and on hyperkinesias when the internal part of the globus pallidus (GPi) is stimulated. ${ }^{8}{ }^{9}$ We treated a patient with a severe, intractable form of Tourette's syndrome by stimulation of the centromedian-parafascicular complex (Ce$\mathrm{Pf})$, the internal part of which is an important associative and limbic relay of the striato-thalamo-cortical pathways, ${ }^{10}$ and the GPi, the anteromedial part of which is a limbic relay for output pathways of the basal ganglia. ${ }^{11}$

\section{METHODS \\ Patients}

Between January 1999 and August 2001, five patients were selected from among the 40 who consulted for Tourette's syndrome. Inclusion criteria were: age $\geqslant 18$ years; severe form of the disease adversely affecting social integration; failure of medical treatments after a trial of at least six months; no cognitive deficits or psychosis; and ability to give informed consent. One patient refused, three asked for a period of reflection, and one accepted after approval of the local ethics committee.

\section{Case report}

A 36 year old woman began to shrug her shoulders and utter sounds when she was 7. At 14 she developed coprolalia and self injurious behaviour (biting her tongue, pulling out her hair). She could not stand frustration and had relational problems suggestive of a borderline personality disorder. Neuroleptic treatment improved her tics for 14 years. She obtained a secretarial qualification but was fired several times because of the severity of her vociferations. At age 28 , she married and had a son who was placed with his grandparents. The patient suffered anxiety and depression, despite various associations of anxiolytics, antidepressants, neuroleptics, and electroconvulsive therapy. Neighbours petitioned to have her evicted from her apartment. She was found guilty of attacking a driver with a metal bar. Facial contractions, retroversion of the eyes, and touching became worse. Bouts of shrieking recurred 15 to 20 times an hour. She stuck her finger in her eye causing lesions of the cornea, burned her breast and nose with cigarette ends, and bit her lips until they bled.

\section{Procedures}

Four quadripolar leads (model 3389, Medtronic) were implanted bilaterally under general anaesthesia in the Ce-Pf of the thalamus and in the limbic territory of the Gpi, guided by magnetic resonance imaging (MRI) and electrophysiological recordings. ${ }^{12}$ Leads were implanted in the right and left thalamus and the right and left GPi. The two thalamic leads were connected to one stimulator (model 7594, Medtronic) and the two pallidal electrodes to another. The locations of the therapeutic electrodes, as determined postoperatively on MRI aligned in three dimensions with the stereotactic atlas, ${ }^{13}$ were shown to be the parafascicular subdivision of the Ce-Pf (fig 1, panels A and B) and the antero-medial part of the GPi (panels $\mathrm{C}$ and $\mathrm{D}$ ). The intensity of stimulation was the greatest that did not elicit side effects at a constant frequency of $130 \mathrm{~Hz}$ and a pulse width of $60 \mu \mathrm{s}$. The severity of tics was assessed one month before surgery, then after surgery with a double blind randomised protocol in five phases: no stimulation, thalamic, pallidal, or sham (no) stimulation, and combined thalamic and pallidal stimulation. JLH set the electrical variables; CK, LM, and BP blindly evaluated the patient. Assessments included an interview of the patient and her entourage, and evaluations of tic severity, ${ }^{14}{ }^{15}$ depression, ${ }^{16}$ anxiety, ${ }^{17}$ impulsivity, ${ }^{18}$ attention, ${ }^{19}$ episodic memory, ${ }^{20}$ working memory, ${ }^{21}$ and flexibility. ${ }^{22}$

\section{RESULTS}

\section{Before surgery}

The tics were not improved by six months of treatment combining loxapine (700 mg/day), pimozide ( $18 \mathrm{mg} /$ day), venlafaxine (300 mg/day), and clonazepam ( $16 \mathrm{mg} / \mathrm{day})$. The patient (weight $81 \mathrm{~kg}$, height $165 \mathrm{~cm}$ ) was irritable, dysphoric, and anxious with cognitive slowing (impaired attention and executive functions; table 1).

\section{Postoperative phase without stimulation}

From the moment the patient awakened after the operation, there was a spectacular improvement in tic severity and self injurious behaviour. She was weaned from neuroleptics over a two month period. One month after the operation, tics and self injurious behaviour reappeared. Three months after the operation, the tics and self injuries were as severe as before implantation of the electrodes. The neuropsychological evaluations remained normal throughout the follow up (table 1).

Abbreviations: DBS, deep brain stimulation; GPi, internal part of the globus pallidus; RVBTS, Rush video based tic scale; YGTSS, Yale global tic severity scale 

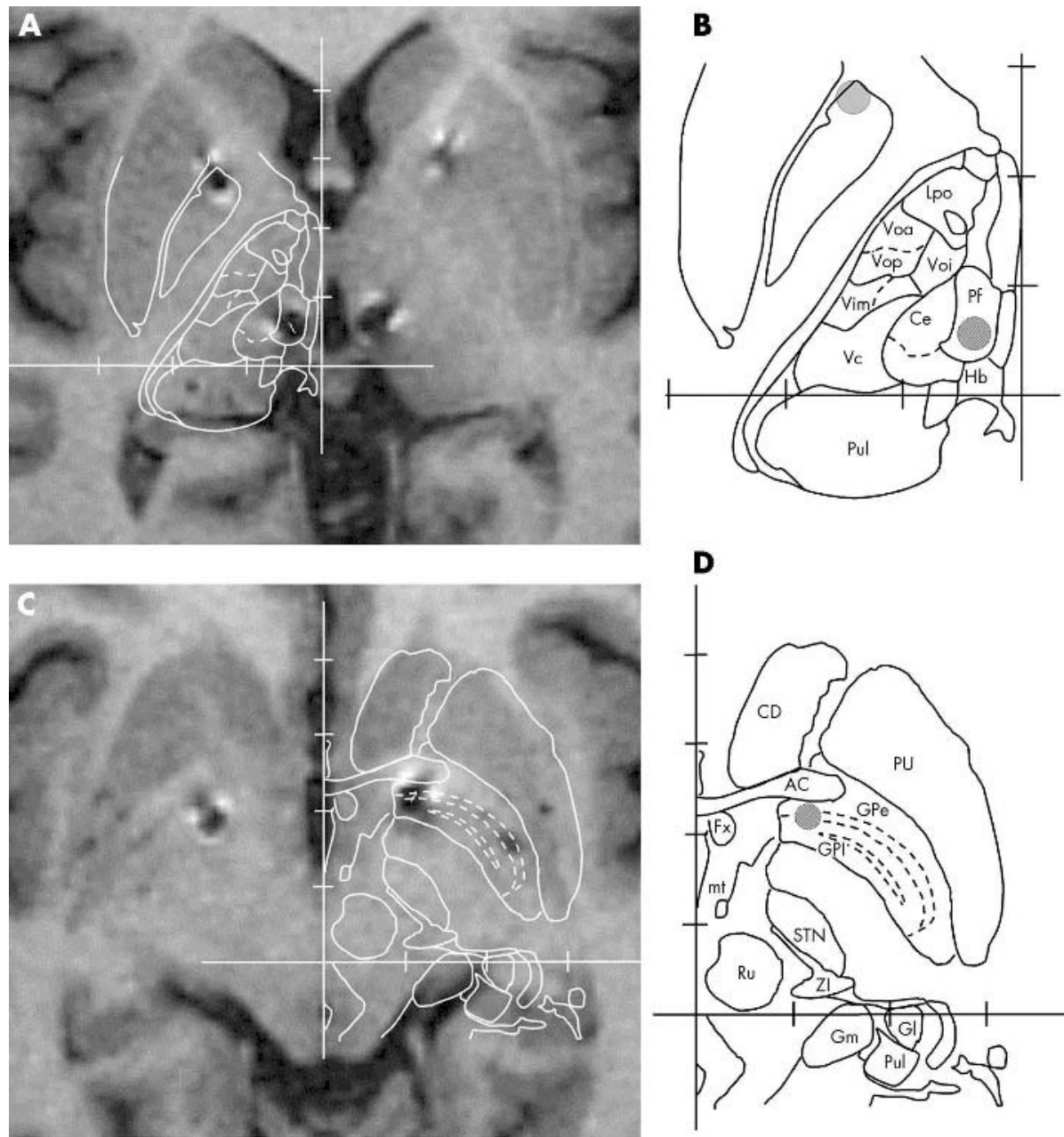

Figure 1 Localisation of the therapeutic contacts in the parafascicular nucleus of the thalamus (panels $A$ and B) and in the pallidum (panels C and D). The thalamic and pallidal structures were delimited by three dimensional alignment of the Schaltenbrand atlas with the axial magnetic resonance images. Hatched circles indicate the location of therapeutic contacts 0 and 1 in the thalamus $(2.9 \mathrm{~mm}$ anterior, $6.1 \mathrm{~mm}$ lateral, $1.9 \mathrm{~mm}$ dorsal to PC point) (Schaltenbrand section: $+3.5 \mathrm{~mm}$ ) (B), and the therapeutic contact $O$ in the pallidum $(22.1 \mathrm{~mm}$ anterior, $11.2 \mathrm{~mm}$ lateral, $3.7 \mathrm{~mm}$ ventral to PC point) (Schaltenbrand section: $-3.5 \mathrm{~mm}$ ) (D). The grey circle (B) indicates the trajectory of the pallidal electrode. $A C$, anterior commissure; $C D$ caudate; $\mathrm{Ce}$, centromedian; Fx, fornix; GPe, external pallidum; GPi, internal pallidum; Gl, lateral geniculate nucleus; Gm, medial geniculate nucleus; $\mathrm{Hb}$, habenula; Lpo, lateropolaris; mt, mamillothalamic tract; Pf, parafascicular nucleus; PU, putamen; Pul, pulvinar; Ru, red nucleus; STN, subthalamic nucleus; Vc, nucleus ventrocaudalis; Vim, nucleus ventrointermedius; Voa, nucleus ventro-oralis anterior; Voi, nucleus ventro-oralis internus; Vop, nucleus ventro-oralis posterior; ZI, zona incerta.

\section{Bilateral thalamic stimulation}

Monopolar stimulation was delivered bilaterally $(1.5 \mathrm{~V}$, ventral contacts 0 and 1 ) in the Ce-Pf (fig 1 , panels A and $\mathrm{B})$. Increasing the voltage induced paraesthesiae in the contralateral half of the tongue. Stimulation through the dorsal contacts (2 and 3 ) induced contraction of the contralateral half of the body. Three weeks later, the self injurious behaviour disappeared and the tics improved, although intermittent coprolalia could be prompted by domestic disputes. The patient bought new undergarments, let her nails grow, and went to nightclubs, but complained of lack of affection from her relatives. She lost weight ( $18 \mathrm{~kg}$ ), but endocrine examinations were normal. After two months, tic severity had decreased by $65 \%$ on the Yale global tic severity scale (YGTSS) ${ }^{14}$ and by $77 \%$ on the Rush video based tic scale (RVBTS). ${ }^{15}$ Mood, anxiety, and impulsivity were improved (table 1).

\section{Bilateral pallidal stimulation}

Monopolar stimulation was delivered bilaterally $(1.5 \mathrm{~V}$, ventral contacts 0 ) in the limbic portion of the GPi (fig 1, panels C and D). Increases in intensity provoked nausea, hypotonia, and anxiety. The tics remained under control, but coprolalia persisted although milder and intermittent. The patient asked for a divorce and wanted to go back to the job she had left two and a half years earlier. Final evaluation confirmed the improvement in tic severity (YGTSS $-65 \%$; RVBTS $-67 \%$ ) and the disappearance of self injurious behaviour, although mood and impulsivity were worse than with thalamic stimulation (table 1). The patient continued to lose weight although she ate normally.

\section{"Sham" stimulation}

One month after the stimulation was stopped, the tics progressively returned, accompanied by panic attacks. Three weeks later, the tics were as severe as before the operation ("before, I was oblivious, now I am lucid and can't stand being this way"). She stuck her finger in her eye, struck her neck causing excoriations at the site of the stimulation cables, and made several attempts to pull out the stimulators. The scores for depression, anxiety, and impulsivity showed little change (table 1). 
Table 1 Evaluation of tic severity, cognitive performance, and psychiatric status before and after neurosurgery

\begin{tabular}{|c|c|c|c|c|c|c|}
\hline & \multirow[b]{2}{*}{$\begin{array}{l}\text { Before } \\
\text { surgery }\end{array}$} & \multicolumn{5}{|c|}{ After surgery: stimulation category } \\
\hline & & None & Thalamic & Pallidal & "Sham" & $\begin{array}{l}\text { Thalamic and } \\
\text { pallidal }\end{array}$ \\
\hline Month: & 0 & 3 & 5 & 7 & 9 & 11 \\
\hline \multicolumn{7}{|l|}{ Tic severity } \\
\hline YGTSS $(/ 100)^{14}$ & 84 & 78 & 30 & 29 & 91 & 34 \\
\hline RVBTS $(/ 20)^{15}$ & 13 & 14 & 3 & 6 & 11 & 3 \\
\hline \multicolumn{7}{|l|}{ Psychiatric evaluation } \\
\hline Depression (MADRS/60) ${ }^{16}$ & 25 & 19 & 10 & 23 & 20 & 17 \\
\hline Anxiety $(\mathrm{BAS} / 60)^{17}$ & 7 & 15 & 8 & 10 & 7 & 15 \\
\hline Impulsivity $(\mathrm{BIS} / 100)^{18}$ & 77 & 66 & 35 & 58 & 61 & 39 \\
\hline \multicolumn{7}{|l|}{ Cognitive evaluation } \\
\hline Attention index $(/ 130)^{19}$ & 76 & 98 & 98 & 98 & 99 & 101 \\
\hline Episodic memory (verbal learning/ & & & & & & \\
\hline 36$)^{20}$ & 14 & - & 30 & 29 & 31 & 31 \\
\hline \multicolumn{7}{|l|}{ Working memory (digit ordering test/ } \\
\hline 105$)^{21}$ & 67 & 86 & 85 & 86 & 86 & 89 \\
\hline Flexibility (trail making B-A) ${ }^{22}$ & 96 & 43 & 28 & 27 & 36 & 30 \\
\hline
\end{tabular}

\section{Bilateral thalamic and pallidal stimulation}

Within 12 hours after the initiation of stimulation, the patient stopped her self injurious behaviour. The tics improved although coprolalia remained moderate. The final evaluation confirmed the abolition of self injuries and the $70 \%$ reduction in tic severity (table 1 ), although some vociferation persisted. The patient said "my life has been transformed...I am glad I had the operation... I feel like a woman again." Her weight stabilised. Twenty four months after the end of the protocol the tics and self injurious behaviour were still improved. The patient divorced, went back to work, and has taken steps to regain custody of her son. Follow up treatment includes regular psychotherapy and antidepressants. Her personality remains borderline, with emotional hypersensitivity, moderate anxiety, phobia, and mood fluctuations.

\section{DISCUSSION}

Continuous high frequency stimulation improved the severity and frequency of tics by about $70 \%$ and self injurious behaviour disappeared when either the thalamus, the GPi, or both were stimulated, indicating that there was no potentiation between the targets. With thalamic stimulation, the patient was less depressed and emotionally unstable than when the GPi was stimulated. Coprolalia improved, but reappeared intermittently whenever there was a loss of mental control. Neither a placebo effect nor spontaneous remission of the disease ${ }^{15}$ is unlikely, as the double blind protocol included two phases without stimulation (postoperative period and sham stimulation period), ${ }^{23}$ and the therapeutic benefit had persisted for 24 months after the operation, despite the withdrawal of neuroleptics.

The clinical improvement in the postoperative phase probably resulted from a transitory lesion caused by placement of the four electrodes, as it waned within a few weeks. ${ }^{6}$ This acute effect contrasted with the delay before tic improvement after thalamic stimulation and before tic worsening during sham stimulation. The reasons for these delays are not known, but they are reminiscent of the delayed effects observed in patients treated by pallidal stimulation for dystonia. ${ }^{9}$

Normalisation of cognitive functions after the operation, in the absence of stimulation, can reasonably be attributed to withdrawal of neuroleptics and confirms that the neurosurgical procedure was not deleterious. The weight loss probably resulted from withdrawal of neuroleptics rather than from stimulation, as all biological test results were normal and the patient's weight eventually stabilised.

The persistence of dysphoria, impulsivity, and difficulty with interpersonal relationships could have resulted from withdrawal of high dose neuroleptics and benzodiazepines, amplification of personality problems, difficulties with social reinsertion after 20 years of affective isolation, or deleterious effects of stimulation, although the absence of psychological change after the postoperative and placebo phases argues against this.

The limbic parts of the GPi and Ce-Pf complex of the thalamus are candidate targets for treatment of severe forms of Tourette's syndrome by high frequency stimulation. These effects are consistent with results obtained in animal experiments on the role of the thalamus and basal ganglia in the occurrence of stereotypies and tics. ${ }^{24}{ }^{25}$ According to these studies, clusters of neurones in the striatum that are important for behavioural selection would, when abnormally active, cause tic production by inhibiting target neurones in output nuclei of the basal ganglia that normally suppress unwanted movements.

High frequency stimulation holds promise for the treatment of other severe forms of Tourette's syndrome. The best target, the thalamus (as previously suggested) ${ }^{37}$ or the GPi (stimulated for the first time), remains to be determined, although other limbic territories of the basal ganglia should also be explored. However, surgical treatment of neuropsychiatric diseases poses serious ethical problems, and there is always the risk of decompensating pre-existing psychiatric disorders. This form of treatment thus requires the adoption of rigorous inclusion criteria and the assurance of follow up by an experienced multidisciplinary team.

\section{ACKNOWLEDGMENTS}

We are indebted to the members of the protocol survey committee: Drs J F Allilaire, J Feingold, and P Cesaro. We thank Dr M Ruberg for reviewing the manuscript, Drs C Behar, B Dubois, N Girault, B Stankoff, and M Vidailhet for care of the patient, and S Monteil for help with the protocol. This protocol was supported by INSERM (contract No 00-008RD331 041) and Medtronic France. 


\section{Authors' affiliations}

J L Hoveto, C Karachi, V Mesnage, M L Welter, J Yelnik, Y Agid, Centre d'Investigation Clinique, INSERM U289, IFR 70, Hôpital de la

Salpêtrière, Paris, France

B Pillon, INSERM EPI 007, Hôpital de la Salpêtrière

L Mallet, A Pelissolo, CNRS UMR7593, Hôpital de la Salpêtrière B Pidoux, Fédération de Neurophysiologie Clinique, Hôpital de la Salpêtrière

D Dormont, Service de Neuroradiologie, Hôpital de la Salpêtrière P Cornu, S Navarro, Service de Neurochirurgie, Hôpital de la Salpêtrière

P Damier, Service de Neurologie, Hôpital Laennec, Nantes, France

Competing interests: none declared

Correspondence to: $\operatorname{Dr}$ Y Agid, Centre d'Investigation Clinique, Hôpital de la Salpêtrière, 47 boulevard de l'Hôpital, 75013 Paris, France; agid@ccr.jussieu.fr

Received 28 April 2004

In revised form 29 November 2004

Accepted 1 December 2004

\section{REFERENCES}

Robertson MM. Tourette syndrome, associated condition and the complexities of treatment. Brain 2000;123:425-62.

2 Leckman JF. Tourette's syndrome. Lancet 2002;360:1577-86.

3 Hassler R, Dieckmann G. Stereotaxic treatment of tics and inarticulate cries or coprolalia considered as motor obsessional phenomena in Gilles de la Tourette's disease. Rev Neurol (Paris) 1970;123:89-100.

4 Lozano AM, Kumar R, Gross RE, et al. Globus pallidus internus pallidotomy for generalized dystonia. Mov Disord 1997;12:865-70.

5 Jankovic J. Tourette's syndrome. N Engl J Med 2001;345:1 184-92.

6 Benabid AL, Benazzouz A, Hoffmann D, et al. Long-term electrical inhibition of deep brain targets in movement disorders. Mov Disord 1998;13:119-25.

7 Visser-Vandewalle V, Temel Y, Boon P, et al. Chronic bilateral thalamic stimulation: a new therapeutic approach in intractable Tourette syndrome. Report of three cases. J Neurosurg 2003;99:1094-100.

8 Beijani BP, Damier P, Arnulf I, et al. Pallidal stimulation for Parkinson's disease. Two targets? Neurology 1997;49:1564-69.
9 Coubes P, Roubertie A, Vayssiere N, et al. Treatment of DYT1-generalised dystonia by stimulation of the internal globus pallidus. Lancet 2000;355:2220-1.

10 Sadikot AF, Parent A, François C. Efferent connections of the centromedian and parafascicular thalamic nuclei in the squirrel monkey: a PHA-L study of subcortical projections. J Comp Neurol 1992;315:137-59.

11 Haber S, Lynd-Balta E, Mitchell SJ. The organisation of descending ventral pallidum projections in monkey. J Comp Neurol 1993;329:111-28.

12 Beijani BP, Dormont D, Pidoux B, et al. Bilateral subthalamic stimulation for Parkinson's disease by using three-dimensional stereotactic magnetic resonance imaging and electrophysiological guidance. J Neurosurg 2000;92:615-25.

13 Yelnik J, Damier P, Bejiani BP, et al. Functional mapping of the human globus pallidus: contrasting effect of stimulation in the internal and external pallidum in Parkinson's disease. Neuroscience 2000;101:77-87.

14 Leckman JF, Riddle MA, Hardin MT, et al. The Yale Global Tic Severity Scale: initial testing of a clinician-rated scale of tic severity. J Am Acad Child Adolesc Psychiatry 1989;28:566-73.

15 Goetz CG, Pappert EJ, Louis ED, et al. Advantages of a modified scoring method for the Rush Video-Based Tic Rating Scale. Mov Disord 1999;14:502-6.

16 Montgomery SA, Asberg M. A new depression scale designed to be sensitive to change. Br J Psychiatry 1979;134:382-9.

17 Tyrer R, Owen RT, Cichetti DV. The brief scale for anxiety: a subdivision of the Comprehensive Psychopathological Rating Scale. I Neurol Neurosurg Psychiatry 1984;47:970-5.

18 Barrat ES, Kent T, Stanford MS. The role of biological variables in defining and measuring personality. In: Ratey JJ, eds. Neuropsychiatry of personality disorders. Cambridge: Blackwell Sciences, 1995:35-49.

19 Wechsler D. Wechsler memory scale-revised manual. San Antonio: The Psychological Corporation, 1987.

20 Brandt J. The Hopkins Verbal Learning Test. Development of a new verbal memory test with 6 equivalent forms. Clin Neuropsychol 1991;5:125-42

21 Cooper JA, Sagar HJ, Jordan N, et al. Cognitive impairment in early, untreated Parkinson's disease and its relationship to motor disability. Brain $1991 ; 114: 2095-122$.

22 Reitan RM. Validity of the trail making test as an indication of organic brain damage. Percept Mot Skills 1958;8:271-6.

23 Feireira JJ. " $\mathrm{N}=1^{\text {" }}$ trials: a possible solution to assess intervention in movement disorders. Mov Disorders 2002;17:S14.

24 Graybiel AM, Canales JJ. The neurobiology of repetitive behaviours: clues to the neurobiology of Tourette syndrome. Adv Neurol 2001;85:123-31.

25 Mink JW. Basal ganglia dysfunction in Tourette's syndrome: a new hypothesis. Pediatr Neurol 2001;25:190-8. 